\title{
Nasal/Oromucosal Solution Dosage Form
}

National Cancer Institute

\section{Source}

National Cancer Institute. NasallOromucosal Solution Dosage Form. NCI Thesaurus.

Code C149692.

Liquid preparation consisting of a solution intended for nasal or oromucosal application. 